\title{
Enhanced Sensitivity to NVP-BEZ235 by Inhibition of p62/SQSTM1 in Human Bladder Cancer KoTCC-1 Cells Both In Vitro and In Vivo
}

\author{
KEITA TAMURA, KYOHEI WATANABE, YUTO MATSUSHITA, HIROMITSU WATANABE, DAISUKE MOTOYAMA, \\ TOSHIKI ITO, TAKAYUKI SUGIYAMA, ATSUSHI OTSUKA and HIDEAKI MIYAKE
}

Department of Urology, Hamamatsu University School of Medicine, Hamamatsu, Japan

\begin{abstract}
Background/Aim: The prognosis of patients with invasive bladder cancer remains poor. The objective of this study was to evaluate the efficacy of NVP-BEZ235 (NVP), a dual PI3K/mTOR inhibitor, combined with the inactivation of p62/SQSTM1 (p62) in a human bladder cancer KoTCC-1 model. Materials and Methods: An expression plasmid with short hairpin RNA targeted against p62 was transfected into KoTCC-1 cells (KoTCC-1/sh-p62). The antitumor effects of NVP on KoTCC-1/sh-p62 were investigated in comparison with those on KoTCC-1 transfected with a control plasmid alone (KoTCC-1/C). Results: KoTCC-1/sh-p62 showed significantly higher sensitivity to NVP than KoTCC-1/C. Treatment of both cell lines with NVP markedly inactivated the PI3K/Akt/mTOR signaling pathway. However, NVP treatment stimulated the autophagic pathway in KoTCC-1/C, but not in KoTCC-1/sh-p62. Furthermore, compared with KoTCC-1/C, NVP treatment induced apoptosis of KoTCC1/sh-p62 cells, which was accompanied by significant downregulation of C-IAP-1 and XIAP as well as upregulation of Bax. Moreover, the in vivo growth of KoTCC-1/sh-p62 tumors was significantly suppressed by treatment with NVP compared to KoTCC-1/C tumors. Conclusion: Inhibition of p62 expression combined with NVP may represent an effective therapeutic approach for patients with invasive bladder cancer.
\end{abstract}

The gold standard treatment for patients with muscleinvasive bladder cancer remains radical cystectomy, with the

This article is freely accessible online.

Correspondence to: Hideaki Miyake, Department of Urology, Hamamatsu University School of Medicine, 1-20-1 Handayama, Higashi-Ku, Hamamatsu 431-3192, Japan. Tel: +81 534352306, Fax: +81 534352305, e-mail: hideakimiyake@hotmail.com

Key Words: NVP-BEZ235, p62/SQSTM1, bladder cancer. 5-year recurrence-free survival rate ranging between 50 and $70 \%$ (1); however, muscle-invasive bladder cancer usually has an aggressive phenotype with a propensity to develop local recurrence as well as distant metastases (2). Furthermore, once recurrence occurs, the prognosis of such patients is extremely poor despite the intensive multimodal treatments being provided (3). Collectively, these findings indicate limitations with respect to further improvements in the prognosis of patients with invasive bladder cancer using conventional therapeutic approaches alone; accordingly, it is necessary to identify optimal therapeutic targets and develop novel molecular-targeted therapy for these patients.

Autophagy is a lysosome-dependent pathway for protein degradation, which is characterized by the formation of double membrane-bound organelles recognized as autophagosomes, and this is markedly activated under metabolic stresses, such as starvation and hypoxia, in order to maintain cellular viability and homeostasis (4). Although dysregulation of the autophagic pathway has been shown to be involved in several pathological processes, including cardiovascular disorders, neurodegeneration and microbial infections, the functional significance of autophagy in carcinogenesis is still controversial. Accumulated evidence suggests the role of autophagy as a prosurvival mechanism to protect neoplastic cells from various types of cellular stress $(5,6)$, whereas the inactivation of autophagyassociated genes under certain conditions has been shown to enhance tumorigenesis (7). p62/SQSTM1 (p62), a multifunctional protein that mediates selective autophagic processes by functioning as an adaptor between the autophagic machinery and its substrates (8), has been demonstrated to integrate molecular events between cancer and autophagy by regulating potential signal transduction pathways during tumor progression (9, 10). Abnormal expression of p62 has been observed in various types of human malignant tumors, such as liver, prostate and breast cancers (11-13); however, the role of p62 in carcinogenesis has not yet been precisely investigated. 
Mammalian target of rapamycin (mTOR) is an evolutionally conserved molecule consisting of multiple proteins, including mTOR complex 1 (mTORC1) and mTORC2, and has a wide variety of important biological functions as one of the major downstream effectors of the phosphoinositide 3-kinase (PI3K)/Akt signaling pathway (14). A number of studies have reported that activation of the mTOR signaling cascade may stimulate the malignant phenotype in various malignant tumors, including bladder cancer $(15,16)$. However, there have been several studies indicating the adaptive induction of autophagy by inhibitors of the PI3K/AKT/mTOR signaling pathway (17), which promotes the survival of cancer cells, limiting their own efficacies.

Collectively, these findings suggest that blockade of autophagic pathway by suppressing p62 expression in combination with PI3K/AKT/mTOR inhibition may efficiently inactivate this tumor survival mechanism and, thus, enhance the cytotoxic activity of PI3K/AKT/mTOR inhibitors against malignant tumors. In this study, therefore, we analyzed changes in the malignant phenotypes of human bladder cancer KoTCC-1 cells both in vitro and in vivo after RNA interference-mediated 'knockdown' of p62, with a focus on the effects of NVP-BEZ235 (NVP), a recently developed dual PI3K/mTOR inhibitor.

\section{Materials and Methods}

Tumor cell lines. The human bladder cancer cell line KoTCC-1 (18) was maintained in RPMI 1640 (Life Technologies Inc., Gaithersburg, MD, USA) supplemented with $10 \%$ fetal bovine serum.

Expression plasmid and transfection to tumor cells. A chemically synthesized oligonucleotide encoding a p62 short-hairpin (sh) RNA (5'-AGACTACGACTTGTGTAGCGTCTGCGAGG-3'), was inserted downstream of the U6 promoter into the pRS expression plasmid (OriGene, Rockville, MD, USA). A control plasmid was constructed by randomizing the sequence of the p62 gene.

Expression plasmids were introduced in cultured KoTCC-1 cells by a liposome-mediated gene transfer method as described previously (19). Briefly, either the control or the shRNA containing plasmid targeted against p62 was added to KoTCC-1 cells following a 30-min pre-incubation with Lipofectamine (Invitrogen, San Diego, CA, USA) and serum-free OPTI-MEM (Life Technologies Inc.). After drug selection in $2 \mu \mathrm{g} / \mathrm{ml}$ puromycin (InVivoGen), each colony was separately expanded to cell line.

Cell growth assay. In order to assess the growth of KoTCC- 1 sublines, $5 \times 10^{3}$ cells from each cell line were seeded in a 96-well plate, allowed to attach, and the number of cells in each well was subsequently evaluated using the Cell Counting Kit-8 (Dojindo, Kumamoto, Japan). The changes in the growth of the KoTCC-1 sublines by NVP treatment were then assessed following a 48-hour incubation.

Western blot analysis. Western blotting was performed as reported previously (19). Briefly, samples consisting of equal amounts of protein $(15 \mu \mathrm{g})$ extracted from the KoTCC-1 sublines cultured in either standard medium or medium containing NVP were electrophoresed on an SDS-polyacrylamide gel and transferred to a nitrocellulose filter. The filters were incubated for 1 hour with antibodies against p62, LC3B, total and phosphorylated (p)-Akt, total and p-mTOR, total and p-4E-BP1, survivin, c-IAP1, XIAP, caspase 3, cleaved caspase 3 (Cell Signaling Technology, Danvers, MA, USA), Bcl-2, Bax, Bcl-xL, or $\beta$-actin (Santa Cruz Biotechnology, Santa Cruz, CA, USA), and were then exposed for $30 \mathrm{~min}$ to a horseradish peroxidase-conjugated anti-mouse or antirabbit IgG antibody (Santa Cruz Biotechnology, Inc., Santa Cruz, CA, USA). Specific proteins were detected with an enhanced chemiluminescence system (Amersham Pharmacia Biotech, Arlington Heights, IL, USA).

Evaluation of in vivo tumor growth. Nude mice (BALB/c-nu/nu male, 6-8 weeks old) used in this study were purchased from Clea Japan (Tokyo, Japan). Animals were maintained according to the National Institutes of Health Guide for the Care and Use of Laboratory Animals. Each experimental group was composed of 5 mice. Approximately $5 \times 10^{6}$ cells suspended in PBS were subcutaneously injected into nude mice. One week after cell injection, mice were randomly assigned to either the intraperitoneal injection of vehicle or $10 \mathrm{mg} / \mathrm{kg}$ NVP 3 times/week for 4 weeks. Each tumor volume was measured with calipers as previously reported (20).

Histopathological assessment of in vivo tumors. Immunohistochemical staining of tumor specimens was carried out as previously described (21). Paraffin-embedded sections were stained using antibodies against p62 and Ki-67 (Cell Signaling Technology) for $1 \mathrm{~h}$, and then incubated with a biotinylated secondary antibody (Santa Cruz Biotechnology, Inc.) for $30 \mathrm{~min}$. After being incubated with an avidin-biotin peroxidase complex, the sections were exposed to diaminobenzidine tetrahydrochloride solution and then stained with methyl green.

Tumor specimens were also examined by TUNEL staining with the in situ cell death detection Kit POD (Roche Applied Science, Indianapolis, IN, USA).

Statistical analysis. The unpaired $t$-test was employed to analyze the differences between the two groups. Statistical analyses were conducted with Statview 5.0 software (Abacus Concepts, Berkley, CA, USA), and $p<0.05$ was considered to indicate statistically significant differences.

\section{Results}

p62 expression in KoTCC-1 sublines. To investigate the effects of p62 expression on the phenotypes of bladder cancer, KoTCC-1 cells were transfected with an expression vector containing shRNA targeted against p62. Several independent clones, were established, and 4 of these clones (KoTCC-1/shp62\#1 to KoTCC-1/sh-p62\#4) were selected for the following studies. Western blotting was performed to compare p62 protein expression levels among the parental KoTCC-1 (KoTCC-1/P), control vector-transfected KoTCC-1 (KoTCC$1 / \mathrm{C}$ ) and the 4 p62 shRNA-transfected clones (KoTCC-1/shp62\#1 to KoTCC-1/sh-p62\#4). As shown in Figure 1a, p62 was abundantly detected in KoTCC-1/P and KoTCC-1/C, whereas the p62 expression levels in the 4 selected clones 
A

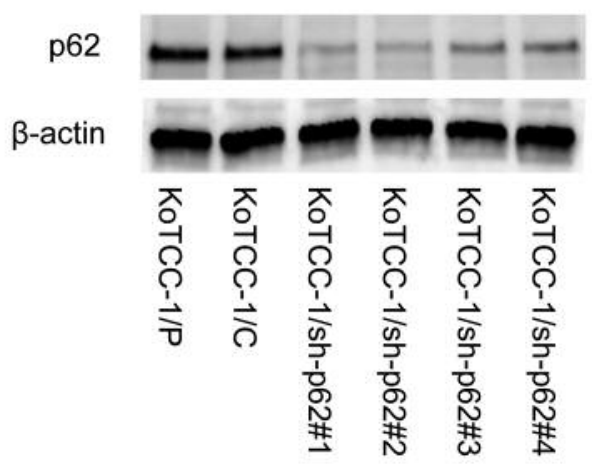

B

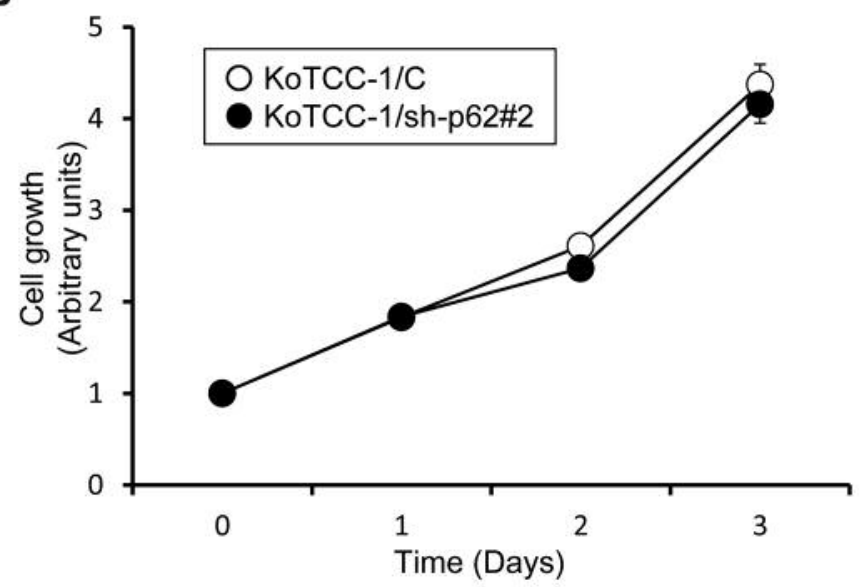

C

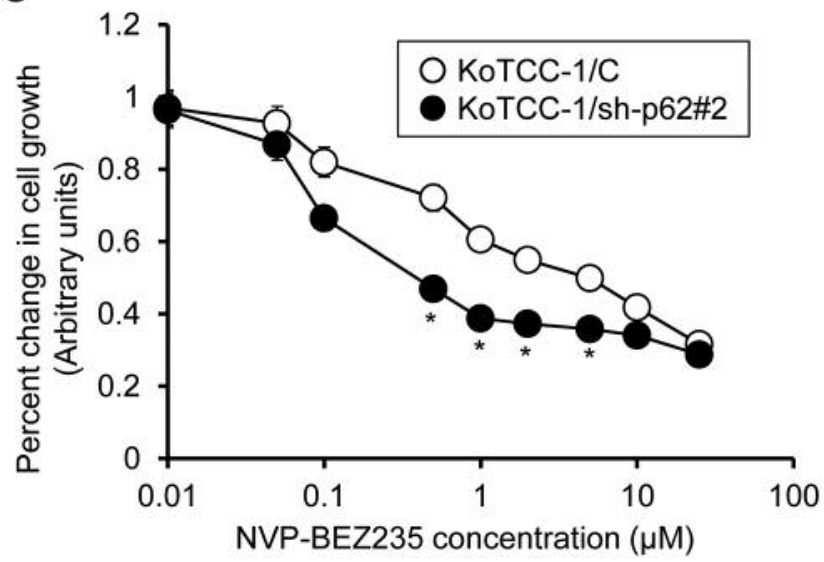

Figure 1. Expression levels of p62 and its effects on cell growth in KoTCC-1 sublines as well as its effect on the sensitivity of these cells to NVPBEZ235. a: Western blotting was performed to analyze the expression levels of p62 and $\beta$-actin in each KoTCC-1 subline (KoTCC-1/P, parental cell line; KoTCC-1/C, control vector-only transfected cell line; KoTCC-1/sh-p62\#1 to \#4, p62 shRNA-transfected cell lines). b: Effects of p62 suppression on the in vitro cell growth of KoTCC-1 sublines. The in vitro proliferation of KoTCC-1/C and KoTCC-1/sh-p62\#2 was measured daily in triplicate in three independent experiments. Bars, SD. c: Effects of p62 suppression on the sensitivity of the KoTCC-1 sublines to NVP-BEZ235. KoTCC-1/C and KoTCC-1/sh-p62\#2 were treated with the indicated doses of NVP-BEZ235. After a 48-h incubation, cell growth was determined from triplicate measurements in three independent experiments. Bars, SD; *significantly different from KoTCC-1/C $(p<0.05)$.

were markedly decreased compared with those in KoTCC-1/P and KoTCC-1/C. Therefore, the results for KoTCC-1/shp62\#2 and KoTCC-1/C alone are subsequently presented.

In vitro growth of KoTCC-1 sublines. The in vitro proliferation patterns of KoTCC-1 sublines cultured in standard medium were initially assessed, and there was no significant difference in growth patterns between KoTCC-1/C and KoTCC-1/shp62\#2 (Figure 1b). In order to determine the inhibitory effect of p62 expression on the sensitivity of KoTCC-1 cells to NVP, KoTCC-1 sublines were treated with several doses of NVP. As shown in Figure 1c, KoTCC-1/sh-p62\#2 showed significantly higher sensitivity to NVP compared with KoTCC-1/C; that is, the $\mathrm{IC}_{50}$ of NVP in KoTCC-1/sh-p62\#2 was approximately $90 \%$ lower than that in KoTCC-1/C.
Expression patterns of key molecules mediating signal transduction and apoptosis in KoTCC-1 sublines. The activity of the PI3K/Akt/mTOR signaling pathway in KoTCC-1 sublines is presented in Figure 2a. Treatment with NVP markedly reduced the expression levels of p-Akt, pmTOR and p-4E-BP1 in both KoTCC-1/C and KoTCC-1/shp62\#2, and no significant differences were noted in the degree of inactivation of the PI3K/Akt/mTOR signaling pathway by NVP treatment between the KoTCC-1 sublines.

Changes in the expression levels of p62 and LC3B in KoTCC-1 sublines following NVP treatment were then examined. Treatment with NVP markedly inhibited p62 expression in KoTCC-1/C, whereas there was no significant change in the levels of p62 expression in KoTCC-1/shp62\#2. Moreover, significant reduction of LC3B-II was 
A

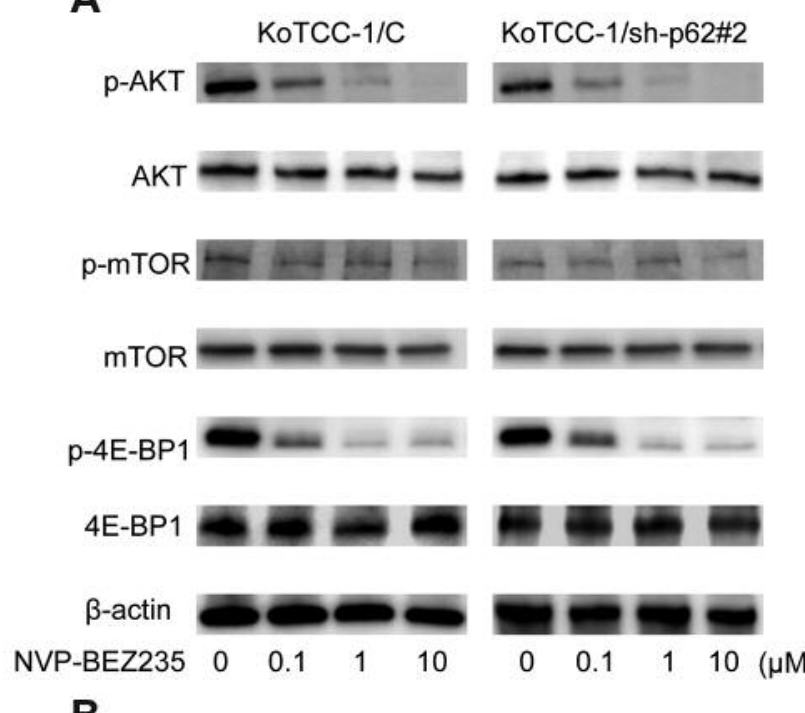

B

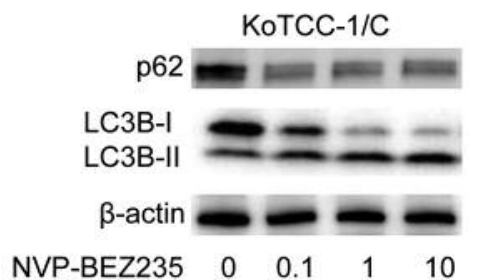

C
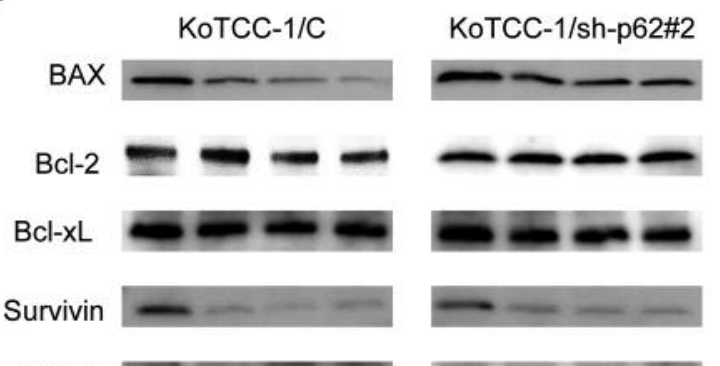

C-IAP-1
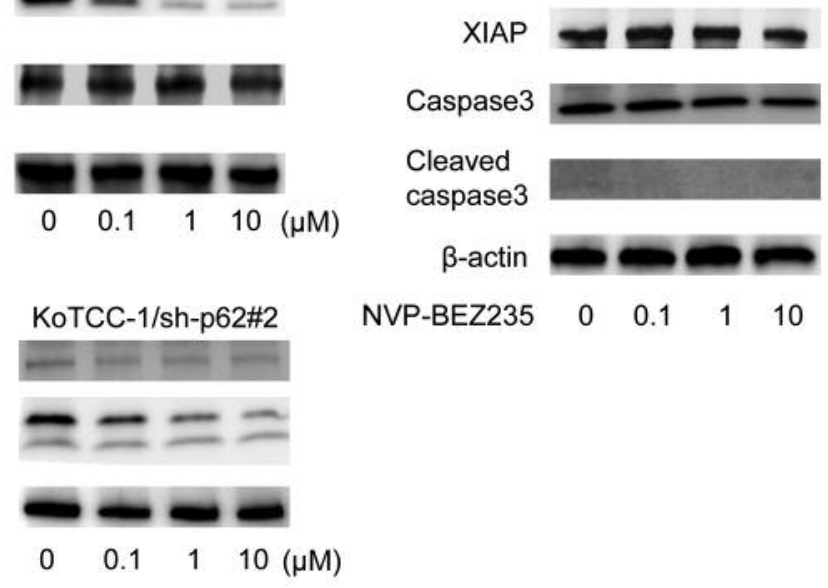

$\begin{array}{lllll}\text { NVP-BEZ235 } & 0 & 0.1 & 1 & 10\end{array}$
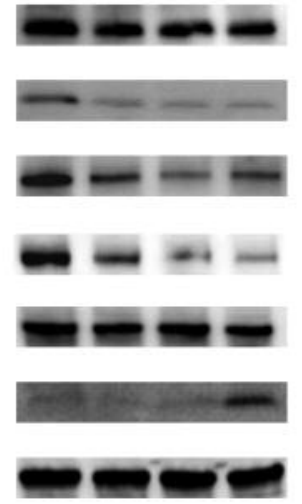

$\begin{array}{lllll}0 & 0.1 & 1 & 10 & (\mu \mathrm{M})\end{array}$

Figure 2. Changes in the expression patterns of key molecules involved in mammalian target of rapamycin (mTOR) signal transduction, autophagy, and apoptosis of KoTCC-1 sublines after the treatment with NVP-BEZ235. a: KoTCC-1 sublines were treated with the indicated concentrations of NVP-BEZ235 for $48 \mathrm{~h}$. Protein was extracted from cultured cells, and evaluated for phosphorylated ( $p$ )-Akt, Akt, p-mTOR, mTOR, $p$-4E-BP1, and $4 E-B P 1$ levels by Western blotting. $b$ : Following the above described treatment schedule, protein was extracted from cultured cells, and analyzed for p62, LC3, and $\beta$-actin levels by western blotting. $c$ : Following the above described treatment schedule, protein was extracted from cultured cells, and analyzed for Bax, Bcl-2, Bcl-xL, Bax, survivin, c-IAP1, XIAP, caspase 3, cleaved caspase 3, and $\beta$-actin levels by western blotting.

observed in in KoTCC-1/sh-p62\#2 compared to KoTCC-1/C (Figure 2b).

The expression patterns of several molecules associated with apoptotic cell death in KoTCC-1 sublines were assessed before and after NVP treatment. As shown in Figure 2c, there were no significant differences in the expression levels of any of the apoptosis-related molecules examined in this study between the KoTCC-1 sublines. Furthermore, treatment with NVP did not result in significant differences in the levels of Bcl-2, Bcl-xL, survivin, or caspase 3 expression between the KoTCC-1 sublines; however, the expression levels of c-IAP1 and XIAP were significantly lower in KoTCC-1/sh-p62\#2 than in KoTCC-1/C, while those of Bax and cleaved caspase 3 were markedly greater in KoTCC-1/sh-p62\#2 than in KoTCC-1/C.

In vivo growth of KoTCC-1 sublines. No significant differences were detected in the in vivo growth patterns between the KoTCC-1 sublines receiving vehicle. However, despite the definite growth inhibition of the KoTCC-1 sublines by the NVP treatment, this effect was significantly weaker on KoTCC-1/C tumors than on KoTCC-1/sh-p62\#2 tumors; that is, the size of KoTCC-1/C tumors was approximately about 2-fold larger than that of KoTCC-1/shp62\#2 tumors 3 weeks after the initiation of the treatment with NVP (Figure 3).

We subsequently evaluated NVP-induced changes in the expression of p62 and the cell growth and apoptotic features in KoTCC-1 sublines in vivo. As shown in Figure 4, p62 expression was not detected in KoTCC-1/sh-p62\#2 irrespective of NVP treatment. However, treatment with NVP significantly reduced the expression of p62 in KoTCC$1 / \mathrm{C}$ tumors. No significant differences were noted in $\mathrm{Ki}-67$ expression patterns between KoTCC-1/C and KoTCC-1/shp62\#2 tumors with or without NVP treatment. In addition, the proportion of TUNEL-positive cells was significantly higher in KoTCC-1/sh-p62\#2 tumors than in KoTCC-1/C tumors after NVP treatment; however, there were no 


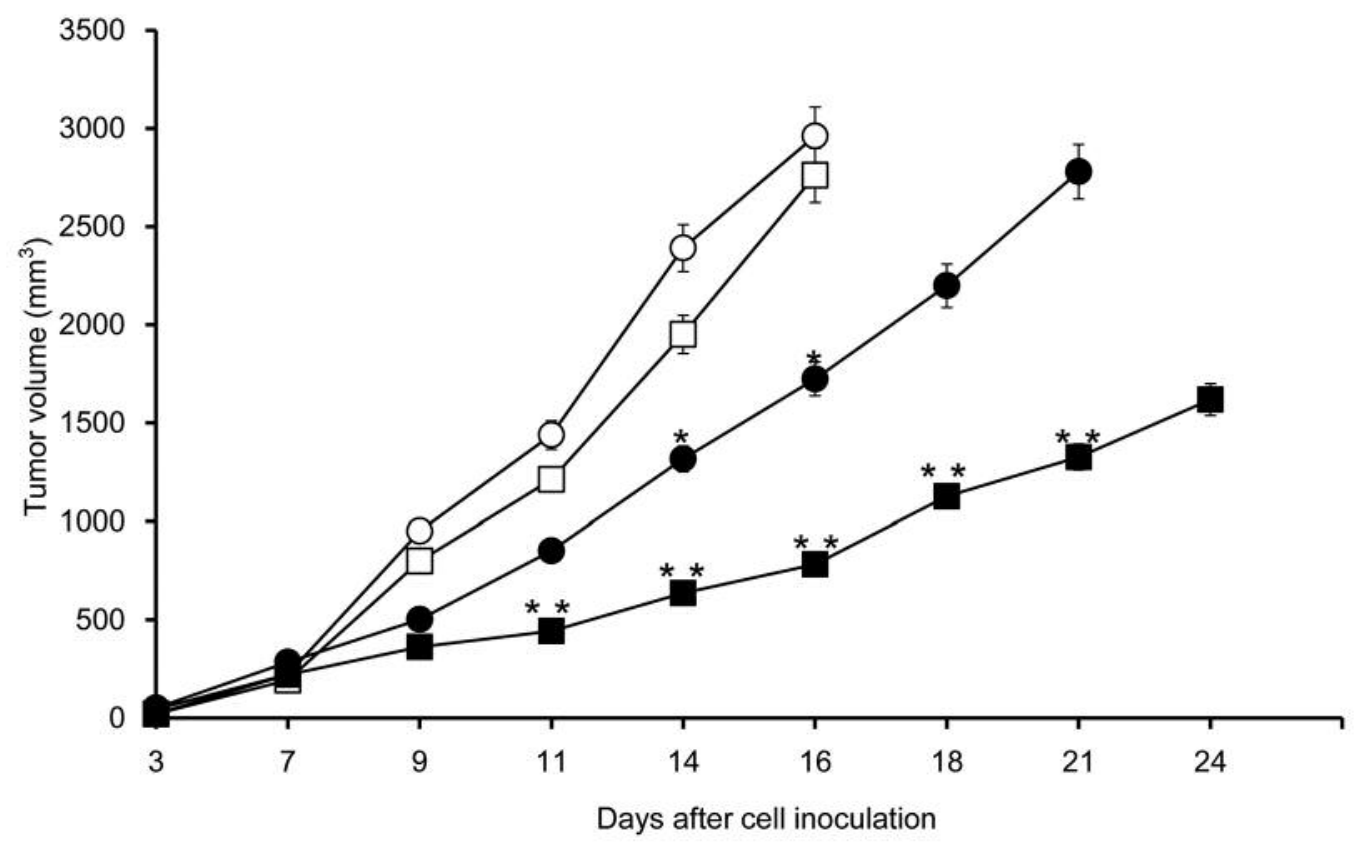

O KoTCC-1/C treated with vehicle

KoTCC-1/C treated with NVP-BEZ235

$\square$ KoTCC-1/sh-p62\#2 treated with vehicle

KoTCC-1/sh-p62\#2 treated with NVP-BEZ235

Figure 3. Effects of the NVP-BEZ235 treatment on tumor growth of KoTCC-1 sublines in nude mice. Ten nude mice were subcutaneously injected with $5 \times 10^{6}$ cells of each KoTCC-1 subline on day 0 , then randomly selected for a treatment with either $10 \mathrm{mg} / \mathrm{kg}$ NVP-BEZ235 or vehicle 3 times per week. Tumor volume was measured using calipers and calculated as length $\times$ width $\times$ depth $\times 0.5236$. Data points represent mean tumor volumes. Bars, $S D$;** and *significantly different from KoTCC-1/C ( $p<0.01$ and $p<0.05$, respectively).

significant differences in the proportion of TUNEL-positive cells between KoTCC-1/sh-p62\#2 and KoTCC-1/C tumors without the NVP treatment.

\section{Discussion}

Owing to the aggressive feature of muscle-invasive bladder cancer, which is characterized by the high probability of disease recurrence after radical cystectomy and extremely unfavorable prognostic outcomes in those developing postoperative disease recurrence (3), it is necessary to establish efficacious therapeutic strategies using novel agents with mechanisms of action different from those currently available in real-world clinical practice. One promising approach is to identify molecular targets mediating the progression of invasive bladder cancer. To date, numerous studies have suggested the important role of the autophagic and $\mathrm{PI} 3 \mathrm{~K} / \mathrm{Akt} / \mathrm{mTOR}$ pathways in the progression of various malignant tumors, including bladder cancer $(22,23)$. However, limited studies are available with respect to the significance of p62, which has been demonstrated to function as a molecular link between cancer and autophagy by regulating major signaling pathways, like the PI3K/Akt/mTOR pathway. Considering these findings, we analyzed the impact of downregulating p62 expression in human bladder cancer KoTCC-1 cells on their malignant phenotypes, with a focus on changes in the activity of the novel dual PI3K/mTOR inhibitor, NVP, both in vitro and in vivo.

Despite the successful establishment of a KoTCC-1 subline in which p62 expression was markedly inhibited, there were no significant differences in proliferative patterns between the KoTCC-1 sublines cultured in standard medium. We then showed identical expression profiles for the major molecules associated with the PI3K/Akt/mTOR pathway and apoptotic cell death between KOTCC-1 sublines cultured in standard media, which suggests no significant differences in proliferative patterns between these sublines. However, whether p62 expression levels modulate the proliferation of cancer cells is still controversial. For example, Ren et al. have reported that the specific knockdown of p62 suppressed the growth of colorectal cancer cells both in vitro and in vivo (24), 


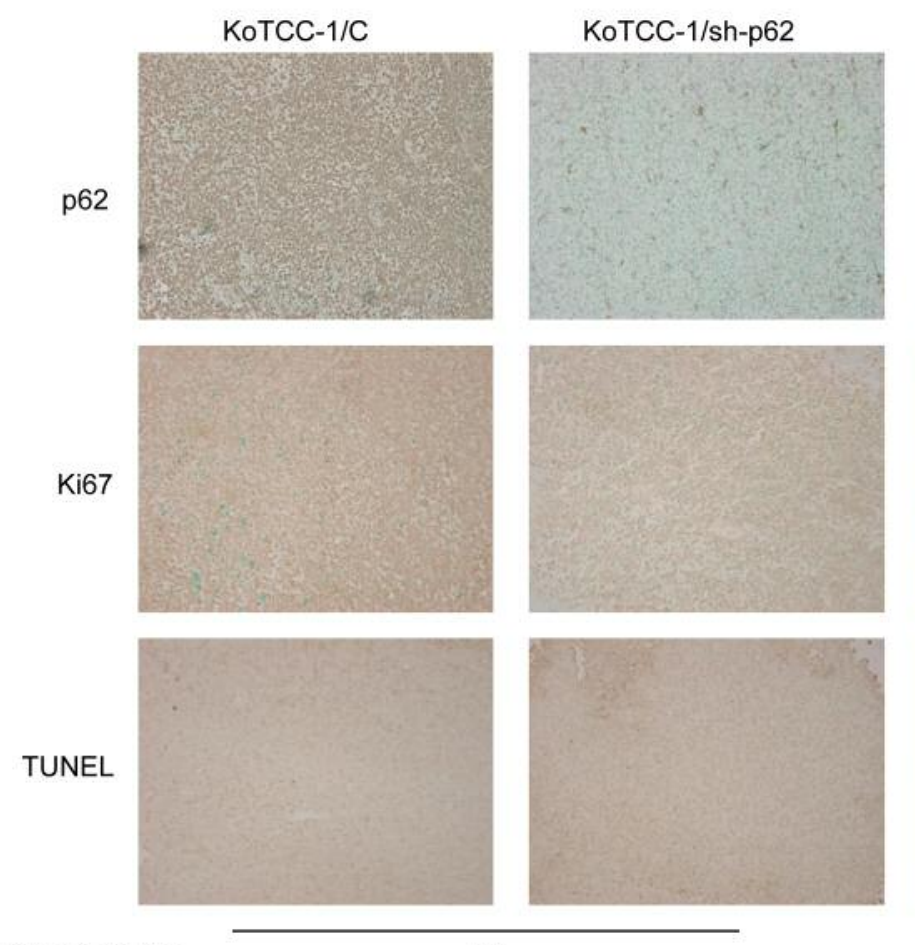

NVP-BEZ235

$(-)$
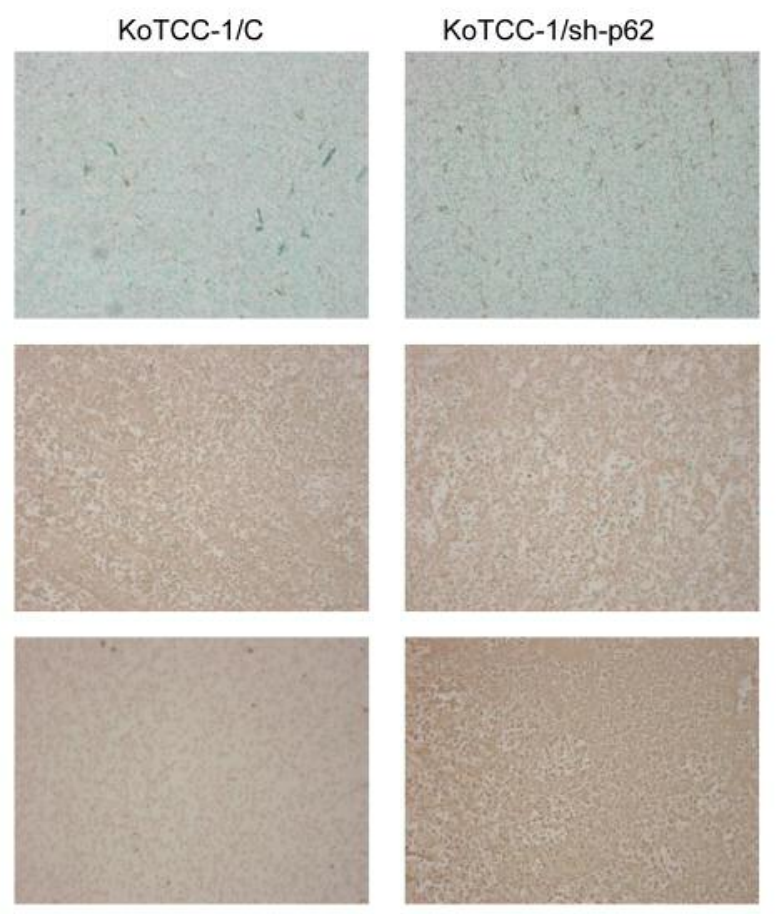

$(+)$

Figure 4. Histopathological study of KoTCC-1 tumors after treatment with NVP-BEZ235. In vivo subcutaneous tumors were harvested from nude mice undergoing treatment with NVP-BEZ235 or vehicle for 4 weeks according to the schedule described in Figure 3. Sections from each tumor tissue were examined by immunohistochemical staining with antibodies against Ki-67 and p62, and TUNEL staining.

whereas Zhou et al. have revealed the lack of significant impact of ampelopsin-induced changes in p62 expression on the growth of breast cancer cells (25). Taken together, these studies and our results suggest that the effect of p62 status on the proliferation of cancer cells may depend on the molecular characteristics of each cancer cell line.

NVP has been shown to inactivate PI3K and mTOR kinases by binding reversibly and competitively to the ATPbinding cleft of these enzymes, and have powerful antitumor effects on several types of malignant tumors (26), including bladder cancer; therefore, its therapeutic utility has been tested in early phase clinical trials. For example, Moon et al. have reported that NVP synergistically enhanced the antitumor activity of cisplatin on cisplatin-resistant bladder cancer cells by suppressing cell cycle progression (27). Based on these outcomes, we assessed whether the antitumor activity of NVP in KoTCC-1 cells was enhanced by inhibiting p62 expression, and showed marked enhancement of the sensitivity of KoTCC-1 cells to NVP by downregulating the p62 expression. There have been some previous studies supporting the findings described above. For example, Chang et al. have revealed that the combined administration of NVP and the autophagy inhibitor 3- methyladenine to hepatocellular carcinoma cells inhibited growth by suppressing the expression of p62 (28). Accordingly, the combination of p62 knockdown with NVP treatment it could be a promising strategy to further improve prognosis of muscle-invasive bladder cancer patients.

It is of interest to investigate the mechanism involved in the enhanced sensitivity of KoTCC-1/sh-p62\#2 to NVP compared to KoTCC-1/C. In the present study, the autophagic pathway was activated in KoTCC-1/C, but not in KoTCC-1/sh-p62\#2, in a dose-dependent manner, by the administration of NVP, while stronger expression of Bax as well as weaker expression of c-IAP-1 and XIAP in KoTCC1/sh-p62\#2 compared to KoTCC-1/C was observed after the NVP treatment. These findings suggest that two potential mechanisms play important roles in the cytotoxic effects of NVP on KoTCC-1/sh-p62\#2, including activation of the proapoptotic pathway by blocking of p62 expression in combination with NVP and suppression of NVP-induced adoptive autophagic pathway stimulated by the inhibition of p62 expression. However, some other mechanisms may also affect this result, since recent studies have indicated the involvement of p62 in a wide variety of molecular events with respect to the regulation of cancer progression $(29,30)$. 
Importantly, administration of NVP to nude mice bearing either KoTCC-1 sublines showed higher inhibition of KoTCC-1/sh-p62\#2 tumors compared to KoTCC-1/C tumors. Furthermore, the in vivo therapeutic efficacy of NVP in KoTCC-1/sh-p62\#2 was more marked than its in vitro efficacy, which could be partially explained by the inhibitory effect of NVP on tumor-associated angiogenesis in addition to the growth of the tumor itself (31). Although it will be required to determine the optimal treatment schedules for NVP in order to maximize its therapeutic activity by avoiding adverse events, the findings of the present study suggest that it will be promising to simultaneously inactivate the PI3K/Akt/mTOR and autophagic pathways to obtain significant inhibition of the growth of invasive bladder cancer.

\section{Conclusion}

Silencing p62 with shRNA interference technique markedly resulted in enhanced sensitivity of KoTCC-1 cells to NVP both in vitro and in vivo; therefore, the inhibition of p62 in combination with NVP could be a promising therapeutic approach for muscle-invasive bladder cancer.

\section{Conflicts of Interest}

The Authors have no conflicts of interest to declare regarding this study.

\section{Authors' Contributions}

Each Author contributed as follows: Study conception and design, Hideaki Miyake; Acquisition of data, Keita Tamura, Kyohei Watanabe, Yuto Matsushita, Hiromitsu Watanabe, Daisuke Motoyama, Toshiki Ito, Takayuki Sugiyama, Atsushi Otsuka; Analysis and interpretation of data, Keita Tamura, Hideaki Miyake; Drafting of manuscript, Keita Tamura, Hideaki Miyake.

\section{Acknowledgements}

No funding or sponsorship was received for this study or publication of this article.

\section{References}

1 Madersbacher S, Hochreiter W, Burkhard F, Thalmann GN, Danuser H, Markwalder R and Studer UE: Radical cystectomy for bladder cancer today-a homogeneous series without neoadjuvant therapy. J Clin Oncol 21: 690-696, 2003. PMID: 12586807. DOI: $10.1200 / \mathrm{JCO} .2003 .05 .101$

2 Stein JP, Lieskovsky G, Cote R, Groshen S, Feng AC, Boyd S, Skinner E, Bochner B, Thangathurai D, Mikhail M, Raghavan $\mathrm{D}$ and Skinner DG: Radical cystectomy in the treatment of invasive bladder cancer: long-term results in 1,054 patients. J Clin Oncol 19: 666-675, 2001. PMID: 11157016. DOI: 10.1200/JCO.2001.19.3.666
3 Pelucchi C, Bosetti C, Negri E, Malvezzi M and La Vecchia C: Mechanisms of disease: The epidemiology of bladder cancer. Nat Clin Pract Urol 3: 327-340, 2006. PMID: 16763645. DOI: $10.1038 /$ ncpuro0510

4 Mizushima N, Levine B, Cuervo AM and Klionsky DJ: Autophagy fights disease through cellular self-digestion. Nature 451: 10691075, 2008. PMID: 18305538. DOI: 10.1038/nature06639

5 Altman BJ, Jacobs SR, Mason EF, Michalek RD, MacIntyre AN, Coloff JL, Ilkayeva O, Jia W, He YW and Rathmell JC: Autophagy is essential to suppress cell stress and to allow BCRAbl-mediated leukemogenesis. Oncogene 30: 1855-1867, 2011. PMID: 21151168. DOI: 10.1038/onc.2010.561

6 Janku F, McConkey DJ, Hong DS and Kurzrock R: Autophagy as a target for anticancer therapy. Nat Rev Clin Oncol 8: 528539, 2011. PMID: 21587219. DOI: $10.1038 /$ nrclinonc.2011.71

7 Mathew R, Karantza-Wadsworth V and White E: Role of autophagy in cancer. Nature reviews. Cancer 7: 961-967, 2007. PMID: 17972889. DOI: $10.1038 / \mathrm{nrc} 2254$

8 Puissant A, Fenouille N and Auberger P: When autophagy meets cancer through p62/SQSTM1. Am J Cancer Res 2: 397-413, 2012. PMID: 22860231.

9 Duran A, Linares JF, Galvez AS, Wikenheiser K, Flores JM, Diaz-Meco MT and Moscat J: The signaling adaptor p62 is an important NF-kappaB mediator in tumorigenesis. Cancer Cell 13: 343-354, 2008. PMID: 18394557. DOI: 10.1016/j.ccr. 2008.02.001

10 Duran A, Amanchy R, Linares JF, Joshi J, Abu-Baker S, Porollo A, Hansen M, Moscat $\mathrm{J}$ and Diaz-Meco MT: p62 is a key regulator of nutrient sensing in the mTORC1 pathway. Mol Cell 44: 134-146, 2011. PMID: 21981924. DOI: 10.1016/j.molcel. 2011.06 .038

11 Kitamura H, Torigoe T, Asanuma H, Hisasue SI, Suzuki K, Tsukamoto T, Satoh M and Sato N: Cytosolic overexpression of p62 sequestosome 1 in neoplastic prostate tissue. Histopathology 48: 157-161, 2006. PMID: 16405664. DOI: 10.1111/j.13652559.2005.02313.x

12 Stumptner C, Heid H, Fuchsbichler, A, Hauser H, Mischinger HJ, Zatloukal K and Denk H: Analysis of intracytoplasmic hyaline bodies in a hepatocellular carcinoma - Demonstration of p62 as major constituent. Am J Pathol 154: 1701-1710, 1999. PMID: 10362795. DOI: 10.1016/S0002-9440(10)65426-0

13 Kim JS, Bae GE, Kim KH, Lee SI, Chung C, Lee D, Lee TH, Kwon IS and Yeo MK: Prognostic significance of LC3B and p62/SQSTM1 expression in gastric adenocarcinoma. Anticancer Res 39: 6711-6722, 2019. PMID: 31810936. DOI: 10.21873/ anticanres. 13886

14 Moschetta M, Reale A, Marasco C, Vacca A and Carratù MR: Therapeutic targeting of the mTOR-signalling pathway in cancer: benefits and limitations. Br J Pharmacol 171: 3801-3813, 2014. PMID: 24780124. DOI: 10.1111/bph.12749

15 Chen Y, Lee CH, Tseng BY, Tsai YH, Tsai HW, Yao CL and Tseng SH: AZD8055 exerts antitumor effects on colon cancer cells by inhibiting mTOR and cell-cycle progression. Anticancer Res 38: 1445-1454, 2018. PMID: 29491070. DOI: 10.21873/ anticanres.12369

16 Nishikawa M, Miyake H, Behnsawy HM and Fujisawa M: Significance of 4E-binding protein 1 as a therapeutic target for invasive urothelial carcinoma of the bladder. Urol Oncol 33: 166.e9-e15, 2015. PMID: 25618298. DOI: 10.1016/j.urolonc. 2014.12.006 
17 Nam HY, Han MW, Chang HW, Kim SY and Kim SW: Prolonged autophagy by MTOR inhibitor leads radioresistant cancer cells into senescence. Autophagy 9: 1631-1632, 2013. PMID: 23989658. DOI: 10.4161/auto.25879

18 Miyake H, Yoshimura K, Hara I, Eto H, Arakawa $\mathrm{S}$ and Kamidono S: Basic fibroblast growth factor regulates matrix metalloproteinases production and in vitro invasiveness in human bladder cancer cell lines. J Urol 157: 2351-2355, 1997. PMID: 9146669.

19 Terakawa T, Miyake H, Furukawa J, Ettinger SL, Gleave ME and Fujisawa M: Enhanced sensitivity to androgen withdrawal due to overexpression of interleukin- 6 in androgen-dependent human prostate cancer LNCaP cells. Br J Cancer 101: 17311739, 2009. PMID: 19844233. DOI: 10.1038/sj.bjc.6605358

20 Miyake H, Yamanaka K, Muramaki M, Hara I and Gleave ME: Therapeutic efficacy of adenoviral-mediated p53 gene transfer is synergistically enhanced by combined use of antisense oligodeoxynucleotide targeting clusterin gene in a human bladder cancer model. Neoplasia 7: 171-179, 2005. PMID: 15802022. DOI: $10.1593 /$ neo.04478

21 Liu B, Miyake H, Nishikawa M and Fujisawa M: Expression profile of epithelial-mesenchymal transition markers in nonmuscle-invasive urothelial carcinoma of the bladder: Correlation with intravesical recurrence following transurethral resection. Urol Oncol 33: 110.e111-e118, 2015. PMID: 25262382. DOI: 10.1016/j.urolonc.2014.08.012

22 Hong SW, Shin JS, Moon JH, Kim YS, Lee J, Choi EK, Ha SH, Lee DH, Chung HN, Kim JE, Kim KP, Hong YS, Lee JL, Lee WJ, Choi EK, Lee JS, Jin DH and Kim TW: NVP-BEZ235, a dual PI3K/mTOR inhibitor, induces cell death through alternate routes in prostate cancer cells depending on the PTEN genotype. Apoptosis 19: 895-904, 2014. PMID: 24652480. DOI: 10.1007/ s10495-014-0973-4

23 Tran AT, Ramalinga M, Kedir H, Clarke R and Kumar D: Autophagy inhibitor 3-methyladenine potentiates apoptosis induced by dietary tocotrienols in breast cancer cells. Eur J Nutr 54: 265-272, 2015. PMID: 24830781. DOI: 10.1007/s00394014-0707-y

24 Ren F, Shu G, Liu G, Zhou J, Yuan L and Zhou J: Knockdown of p62/sequestosome 1 attenuates autophagy and inhibits colorectal cancer cell growth. Mol Cell Biochem 385: 95-102, 2014. PMID: 24065390. DOI: 10.1007/s11010-013-1818-0

25 Zhou Y, Liang X, Chang H, Shu F, Wu Y, Zhang T, Fu Y, Zhang $\mathrm{Q}$, Zhu JD and Mi M: Ampelopsin-induced autophagy protects breast cancer cells from apoptosis through Akt-mTOR pathway via endoplasmic reticulum stress. Cancer Sci 105: 1279-1287, 2014. PMID: 25088800. DOI: $10.1111 /$ cas. 12494
26 Maira SM, Stauffer F, Brueggen J, Furet P, Schnell C, Fritsch C, Brachmann $S$, Chène $P$, De Pover A, Schoemaker K, Fabbro D, Gabriel D, Simonen M, Murphy L, Finan P, Sellers W and García-Echeverría C: Identification and characterization of NVPBEZ235, a new orally available dual phosphatidylinositol 3kinase/mammalian target of rapamycin inhibitor with potent in vivo antitumor activity. Mol Cancer Ther 7: 1851-1863, 2008. PMID: 18606717. DOI: 10.1158/1535-7163.MCT-08-0017

27 Moon du G, Lee SE, Oh MM, Lee SC, Jeong SJ, Hong SK, Yoon CY, Byun SS, Park HS and Cheon J: NVP-BEZ235, a dual $\mathrm{PI} 3 \mathrm{~K} / \mathrm{mTOR}$ inhibitor synergistically potentiates the antitumor effects of cisplatin in bladder cancer cells. Int J Oncol 45: 10271035, 2014. PMID: 24969552. DOI: 10.3892/ijo.2014.2505

28 Chang Z, Shi G, Jin J, Guo H, Guo X, Luo F, Song Y and Jia X: Dual PI3K/mTOR inhibitor NVP-BEZ235-induced apoptosis of hepatocellular carcinoma cell lines is enhanced by inhibitors of autophagy. Int J Mol Med 31: 1449-1456, 2013. PMID: 23588698. DOI: 10.3892/ijmm.2013.1351

29 Duran A, Linares JF, Galvez AS, Wikenheiser K, Flores JM, Diaz-Meco MT and Moscat J: The signaling adaptor p62 is an important NF-kappaB mediator in tumorigenesis. Cancer Cell 13: 343-354, 2008. PMID: 18394557. DOI: 10.1016/j.ccr. 2008.02 .001

30 Son YO, Pratheeshkumar P, Roy RV, Hitron JA, Wang L, Zhang $\mathrm{Z}$ and Shi X: Nrf2/p62 signaling in apoptosis resistance and its role in cadmium-induced carcinogenesis. J Biol Chem 289: 28660-28675, 2014. PMID: 25157103. DOI: 10.1074/jbc. M114.595496

31 Awasthi N, Yen PL, Schwarz MA and Schwarz RE: The efficacy of a novel, dual PI3K/mTOR inhibitor NVP-BEZ235 to enhance chemotherapy and antiangiogenic response in pancreatic cancer. J Cell Biochem 113: 784-791, 2012. PMID: 22020918. DOI: $10.1002 /$ jcb. 23405 